\title{
A multi-center blinded study on the efficiency of phenotypic screening methods to detect glycopeptide intermediately susceptible Staphylococcus aureus (GISA) and heterogeneous GISA (h-GISA)
}

\author{
Andreas Voss*1,2, Johan W Mouton², Erika P van Elzakker ${ }^{3}$, Ron G Hendrix ${ }^{4}$, \\ Wil Goessens ${ }^{5}$, Jan A Kluytmans ${ }^{6}$, Paul F Krabbe ${ }^{7}$, Han J de Neeling, \\ Jacobus H Sloos ${ }^{9}$, Nefise Oztoprak ${ }^{10}$, Robin A Howe ${ }^{11}$ and \\ Timothy R Walsh ${ }^{11}$
}

Address: ${ }^{1}$ Radboud University Nijmegen Medical Centre, Nijmegen University Centre of Infectious Diseases, The Netherlands, ${ }^{2}$ CanisiusWilhelmia Hospital, Department of Medical Microbiology and Infectious Diseases, Nijmegen, The Netherlands, ${ }^{3}$ Medical Centre Delft (SSDZ), Department of Medical Microbiology, The Netherlands, ${ }^{4}$ Regional Laboratory Twente en Achterhoek, Department of Medical Microbiology, Enschede, The Netherlands, ${ }^{5}$ University Medical Centre Erasmus, Department of Medical Microbiology, Rotterdam, The Netherlands, ${ }^{6} \mathrm{Amphia}$ Ziekenhuis, Department of Medical Microbiology, Breda, The Netherlands, ${ }^{7}$ Radboud University Nijmegen Medical Centre, Department of Medical Technology Assessment, The Netherlands, ${ }^{8}$ RIVM, National Institute of Public Health, Bilthoven, The Netherlands, ${ }^{9}$ Regional Laboratory, Medical Center Alkmaar, Alkmaar, The Netherlands, ${ }^{10}$ Zonguldak Karaelmas University Department of Infectious Diseases and Clinical Microbiology, Zonguldak, Turkey and ${ }^{11}$ Bristol Centre for Antimicrobial Research \& Evaluation, Department of Medical Microbiology, Southmead Hospital, Bristol, UK

Email: Andreas Voss* - a.voss@cwz.nl; JohanW Mouton - mouton@cwz.nl; Erika P van Elzakker - elzakker@rdgg.nl;

Ron G Hendrix - r.hendrix@labmicta.nl; Wil Goessens - w.goessens@erasmusmc.nl; Jan A Kluytmans - jankluytmans@gmail.com; Paul F Krabbe - p.krabbe@mta.umcn.nl; Han J de Neeling - han.de.neeling@rivm.nl; Jacobus H Sloos - j.h.sloos@mca.nl; Nefise Oztoprak - nefiseoztoprak@yahoo.com; Robin A Howe - HoweRA@Cardiff.ac.uk; Timothy R Walsh - WalshTR@Cardiff.ac.uk

* Corresponding author

Published: 24 September 2007

Annals of Clinical Microbiology and Antimicrobials 2007, 6:9 doi:I0.1 I86/I476-07| I-6-9
Received: 17 August 2007

Accepted: 24 September 2007

This article is available from: http://www.ann-clinmicrob.com/content/6/1/9

(c) 2007 Voss et al; licensee BioMed Central Ltd.

This is an Open Access article distributed under the terms of the Creative Commons Attribution License (http://creativecommons.org/licenses/by/2.0), which permits unrestricted use, distribution, and reproduction in any medium, provided the original work is properly cited.

\begin{abstract}
Backgrounds: To determine the true incidence of hGISA/GISA and its consequent clinical impact, methods must be defined that will reliably and reproducibly discriminate these resistant phenotypes from vancomycin susceptible $S$. aureus (VSSA).

Methods: This study assessed and compared the ability of eight Dutch laboratories under blinded conditions to discriminate VSSA from hGISA/GISA phenotypes and the intra- and inter-laboratory reproducibility of agar screening plates and the Etest method. A total of 25 blinded and unique strains (I0 VSSA, 9 hGISA and 6 GISA) were categorized by the PAP-AUC method and PFGE typed to eliminate clonal duplication. All strains were deliberately added in quadruplets to evaluate intralaboratory variability and reproducibility of the methods. Strains were tested using three agar screening methods, Brain Heart Infusion agar (BHI) $+6 \mu \mathrm{g} / \mathrm{ml}$ vancomycin, Mueller Hinton agar (MH) $+5 \mu \mathrm{g} / \mathrm{ml}$ vancomycin and $\mathrm{MH}+5 \mu \mathrm{g} / \mathrm{ml}$ teicoplanin) and the Etest macromethod using a 2 McFarland inoculum.
\end{abstract}

Results and Discussion: The ability to detect the hGISA/GISA phenotypes varied significantly between methods and phenotypes. BHI vancomycin and $\mathrm{MH}$ vancomycin agar screens lacked the 
ability to detect hGISA. The MH teicoplanin agar screen was more sensitive but still inferior to Etest that had a sensitivity of $98.5 \%$ and $99.5 \%$, for hGISA and GISA, respectively. Intra- and interlaboratory reproducibility varied between methods with poorest performance seen with $\mathrm{BHI}$ vancomycin.

Conclusion: This is the first multi-center blinded study to be undertaken evaluating various methods to detect GISA and hGISA. These data showed that the ability of clinical laboratories to detect GISA and hGISA varied considerably, and that screening plates with vancomycin have a poor performance in detecting hGISA.

\section{Background}

Since the advent of the first glycopeptide intermediately susceptible $S$. aureus (GISA) and its heterogeneous variant (hGISA) in 1997, debate still ensues as to their clinical significance [1-9]. This perhaps has been compounded by the discovery of the vanA-mediated glycopeptide resistant $S$. aureus from the US where the glycopeptide minimum inhibitory concentrations are demonstrably higher and testing issues are also present, but less problematic $[10,11]$. However, the detection of GISA and hGISA are hampered by the insensitivity of the basic format of standard methods for capturing these phenotypes. Seven years after the publication of Mu50 (GISA) and Mu3 (hGISA), very little has been resolved as to which methods are the most reliable and reproducible. This in part is due to the different definitions ascribed to GISA and hGISA that are subject to methodological differences and variations in (clinical) breakpoints and cut-off values [12,13]. Whilst GISA has a more "homogeneous" resistant population resulting in a higher, stable (thus more reproducible) vancomycin MIC, h-GISA expresses the resistance at approximately $1 / 10^{6}$ of the native population thereby eluding detection by conventional methods. However, strains testing positive by these methods share phenotypic characteristics such as a thickened looser cross-linked cell wall with glycopeptide intermediate $S$. aureus (GISA) [14-16]. Thus, it is possible that hGISA and GISA strains represent the extremes of a common phenotype conferring reduced susceptibility to glycopeptides.

Controlled studies have shown that GISA strains inhibited by a higher vancomycin MIC value are more frequently associated with clinical failures than VSSA. In contrast, the clinical relevance of h-GISA remains controversial. However, numerous case reports have associated hGISA with a poor response to glycopeptide therapy but studies of their clinical significance have been hampered by difficulties in detecting these strains in the diagnostic laboratory and a lack of confirmatory tests. However, a recent observational study comparing the clinical features of bacteraemia due to hGISA (defined by population analysis profiles - area under the curve [PAP-AUC ratio]) and fully vancomycin susceptible MRSA found that hGISA infection was associated with significantly longer time to defer- vescence (mean 35 vs 2.9 days), and duration of bacteraemia (mean 35 vs. 6.4 days), and a non-significant increase in length of hospital stay (107 vs 37 days) [17]. Clinical failure of vancomycin treatment (defined as fever and bacteraemia $>7$ days into vancomycin therapy) occurred in $100 \%(5 / 5)$ hGISA cases compared to $2.1 \%$ (1/48) MRSA cases.

Studies of the prevalence of GISA and hGISA have also suffered from various non-standardized methods for detection and confirmation of these strains so that intercountry frequencies differ significantly. These differences may reflect genuine geographical variation but are likely to be due to methodological inconsistencies. To facilitate the detection of GISA and in particular hGISA, methods have been proposed where media, inoculum, and period of incubation have been altered [5,18-20]. These include the antibiotic gradient plate, agar screening plates, Etest, population studies (PS) and population analysis profiles - area under the curve assays (PAP-AUC) [21,22]. The most reliable of these is the PAP-AUC; however, it is both specialized and labor intensive. The implementation of these methods for detecting resistance will depend on their intra- and inter-laboratory reproducibility. We describe herein the first multi-center blinded study comparing the reliability of four screening methods in an eight-center setting; namely, to assess and compare the inter-laboratory reproducibility of three "agar screening plates" and the Etest "macromethod" in discriminating VSSA from hGISA/GISA phenotypes.

\section{Methods \\ GISA and $h$-GISA strains}

The study included an international collection of 25 unique strains composed of 10 VSSA, 9 hGISA and 6 GISA as determined by PAP-AUC ratio as previously described [22]. The strains used represented the five major hospital lineages as previously described [6]. All strains were deliberately added in quadruplets to evaluate intra-laboratory variability and reproducibility of the methods. Each of the eight participating laboratories received 100 "numbered" isolates. Investigators were blinded with regard to the isolates' phenotype and were unaware that the collection contained replicates. 


\section{Agar antibiotic screening plates}

Laboratories were asked to perform three screening methods. The first utilized Brain Heart Infusion (BHI) (Becton Dickinson, Cockeysville, Md.) agar supplemented with 6 $\mu \mathrm{g} / \mathrm{ml}$ of vancomycin (BHI-van) [16]. The other two agarscreening media were prepared in a similar manner as the BHI medium. All media were centrally supplied. They consisted of Mueller Hinton agar ( $\mathrm{MH}$ - Becton Dickinson, Cockeysville, Md.) with vancomycin (5 $\mu \mathrm{g} / \mathrm{ml})$ (MH-van) and with teicoplanin $(5 \mu \mathrm{g} / \mathrm{ml})$ (MH-teico), respectively $[19,23]$. Inoculum was prepared as previously described. $10 \mu \mathrm{l}$ of the inoculum suspension was used for spot inoculation of the agar screening plates. Plates were incubated at $35^{\circ} \mathrm{C}$ for 18 and 40 hours. Growth was reported after both 24 and 48 hours.

\section{Etest macromethod}

The Etest macromethod for hGISA/GISA testing as recommended by the manufacturer was used as previously described [20]. Essentially, numerous isolated colonies from an overnight agar culture were suspended into $\mathrm{MH}$ broth to achieve a turbidity corresponding to 2 McFarland. $100 \mu \mathrm{l}$ of the inoculum suspension were pipetted onto a standard BHI agar plate and streaked out evenly and then left to dry completely. Etest vancomycin and teicoplanin MIC gradient strips (AB BIODISK, Solna, Sweden) were then applied and the plates incubated at $35^{\circ} \mathrm{C}$ and read at 24 and 48 hours independently by two technicians. The point of complete inhibition of all growth, including hazes, microcolonies and isolated colonies in the inhibition ellipse, was used as the end-point. Etest macromethod results were interpreted as positive for hGISA/GISA when both vancomycin and teicoplanin modified MICs were $\geq 8$ or when the teicoplanin MIC alone was $\geq 12$. Etest MIC values that fell in between dilutions e.g. $6 \mu \mathrm{g} / \mathrm{ml}$ were not rounded up.

\section{Calculation of sensitivity and specificity}

All results were entered into SPSS [24]. The sensitivity (= the probability that a "positive" case is correctly classified, thus the false negative rate, and the specificity (= the probability that a "negative" case is correctly classified, or the false positive rate) were calculated. Furthermore, Cohen's kappa was calculated as a measure that expresses the agreement between the evaluations of two raters when both are rating the same object. A value of 1 indicates perfect agreement. A value of 0 indicates that agreement is no better than chance.

\section{Results and discussion Instability of h-GISA phenotype}

All GISA and hGISA strains that consistently gave a VSSA phenotype yet were originally categorized as a GISA/ hGISA as judged by PAP-AUC (ratio >0.9), were re-sent to the central laboratory to re-confirm their original PAP-
AUC profile. Two strains (a Norwegian hGISA and a French hGISA) had lost their ability to express the heterogeneous form of the resistance (PAP-AUC ratios of 0.82 and 0.75 , respectively) for vancomycin and were retrospectively excluded from the study. All GISA strains maintained their resistant phenotype.

\section{Specificity and sensitivity}

The sensitivity and specificity of agar screening methods and the Etest macromethod are displayed in table 1. The specificity (i.e. no false positives) of all test methods to define VSSA varied considerably. MH-van and BHI-van gave unacceptable values of $58.7 \%$ and $68.4 \%$, respectively. In contrast, both $\mathrm{MH}$-teico and Etest macromethod gave acceptable values of $92.1 \%$ and $93.3 \%$, respectively. The ability of each method to detect GISA and hGISA also varied considerably (Table 1). The GISA isolates (as defined by conventional MIC methodology) were virtually all detected by Etest (sensitivity of 99.5\%). The agar screening methods varied from one another markedly, the highest sensitivity was MH-teico (95.8\%), followed by BHI-van (85.9\%) and the lowest was MH-van (50.8\%). Predictably, the ability of each method to detect h-GISA varied immensely. Sensitivity values for Etest, MH-teico, BHI-van and MH-van were 98.5\%, 85.0\%, 4.5\% and $1.0 \%$, respectively. Whilst the ability of Etest, and to a lesser degree $\mathrm{MH}$-teico, to detect hGISA maybe regarded as acceptable, the sensitivity values for BHI-van and $\mathrm{MH}$ van are clearly not.

The inter-laboratory reproducibility of the methods as calculated by Cohen's kappa on the quadruplicate samples of each strain is depicted in Table 2. Cohen's kappa values could not be calculated for MH-van for VSSA, MH-van for hGISA and Etest for GISA due to unbalanced variance of components. The inter-laboratory reproducibility for VSSA was acceptable for MH-teico (0.80) and Etest (0.70), and poor for BHI-van (0.30). For h-GISA, BHI-van also performed poorly (0.05) compared to MH-teico (0.93) and Etest (0.88). The reproducibility to detect GISA was

Table I: Sensitivity and specificity of agar screening plates and the Etest macromethod

\begin{tabular}{lcccc}
\hline & \multicolumn{4}{c}{ correctly identified (\%) } \\
\cline { 2 - 5 } & BHI-van & MH-van & MH-teico & Etest \\
\hline $\begin{array}{l}\text { Overall } \\
\begin{array}{l}\text { Sensitivity } \\
\text { Overall }\end{array}\end{array}$ & 44.4 & 25.3 & 90.3 & 99.0 \\
$\begin{array}{l}\text { Specificity } \\
\text { h-GISA }\end{array}$ & 68.4 & 58.7 & 92.1 & 93.3 \\
$\begin{array}{l}\text { Sensitivity } \\
\text { GISA }\end{array}$ & 4.5 & 1.0 & 85.0 & 98.5 \\
Sensitivity & 85.9 & 50.5 & 95.8 & 99.5 \\
\hline
\end{tabular}


Table 2: Inter-laboratory reproducibility of agar screening plates and Etest macromethod

\begin{tabular}{lcccc}
\hline & BHl-van & MH-van & MH-teico & Etest \\
\hline VSSA & 0,29 & $*$ & 0,80 & 0,70 \\
h-GISA & 0,05 & $*$ & 0,93 & 0,88 \\
GISA & 0,70 & 0,91 & 0,89 & $*$ \\
All isolates & 0,97 & 0,95 & 0,96 & $*$ \\
\hline
\end{tabular}

$*=$ unable to estimate due to unbalanced variance components

generally better, namely 0.70 for BHI-van, 0.91 for MHvan, and 0,89 for MH-teico. The overall intra-laboratory reproducibility was predictably high with values of 0.97 , 0.95 and 0.96 for BHI-van, MH-van and MH-teico, respectively.

The marked paucity in controlled studies evaluating the clinical outcome of patients infected with either hGISA or GISA may be due to the difficulty in detecting these resistant phenotypes. Much debate still ensues regarding the clinical significance of GISA and especially hGISA and which methods are best employed to detect them. Methods available vary considerably both at an intra- and intercountry level. For example in the USA, BHI agar screen containing $6 \mu \mathrm{g} / \mathrm{ml}$ of vancomycin is commercially available yet teicoplanin is not available, partly because the drug is not licensed for clinical use in the US. Consequently, most laboratories in the US will preferentially use BHI-van $(6 \mu \mathrm{g} / \mathrm{ml})$ whereas European laboratories will prefer MH-teico $(5 \mu \mathrm{g} / \mathrm{ml})$ or BHI with $4 \mu \mathrm{g} / \mathrm{ml}$ vancomycin $[2,23]$. Inevitably, the variation in prevalence of hGISA and GISA from these regions may reflect the performance of the methodologies employed rather than the true incidence of the resistance. It is well known that both hGISA and GISA are physiologically different from VSSA, possessing a thicker, less cross-linked cell wall and are usually nutritionally deficient and considerably slower growing [8]. Accordingly, standard antimicrobial susceptibility testing methods are not often appropriate in detecting these phenotypes and studies with richer media, heavier inoculum (to detect the low frequency resistance of $1 / 10^{6}$ ) and extended period of incubation have been documented as alternative testing conditions. However, such non-standardized methods are difficult to control and very few studies have been undertaken examining their inter- and intra-laboratory variability.

This is the first multi-center blinded hGISA/GISA study and has highlighted some of strengths and potential deficiencies within the methodological protocol. For instance, on re-evaluating two strains that gave drastically out-lying results, two hGISA had "lost" their resistance and reverted to VSSA on being transferred from the UK to the Netherlands clearly demonstrating the instability of some strains belonging to the hGISA phenotype. The methods chosen for this study are those that have been proposed by various groups as an initial screen to detect hGISA/GISA. The phenotypic categorization of the strains used in this study was based on PAP-AUC, a specialized method chosen by the referral laboratory that is specifically designed to detect resistant sub-populations that exist with hGISA. Perhaps not surprisingly, the screening methods chosen for this study (agar screening plates and Etest macromethod) varied considerably in their ability to detect hGISA and GISA as judged by the participating laboratories. The poorest performing method under these conditions was MH-van $(5 \mu \mathrm{g} / \mathrm{ml})$ with a sensitivity of $25.3 \%$ and a specificity of $58.7 \%$. Whilst, BHI-van $(6 \mu \mathrm{g} / \mathrm{ml})$ performed better, its sensitivity (44.4\%) and specificity $(68.4 \%)$ should be deemed as sub-optimal and caution should be advised when using this screening method. Both Etest macromethod and MH-teico performed very well with a sensitivity of $99.0 \%$ and $90.3 \%$, respectively. Although most methods could detect the higher and more homogeneous level of resistance in GISA (apart from MHvan), the biggest variation between the methods arose in detecting hGISA. This in part may be explained by the difference in inoculum and why using a higher inoculum ( 2 McFarland) proves the rate of detecting the $1 / 10^{6}$ of the cells expressing this type of resistance.

The inter-laboratory variability of these methods varied enormously. The poorest of these was BHI-van that gave a Cohen's kappa value of 0.05 and 0.7 for hGISA and GISA, respectively. Comparatively, the other methods did much better and could be regarded as acceptable. The inter-laboratory variation of a screening method is vitally important if routine microbiology laboratories are going to be encouraged to use such methods. Both MH-teico and Etest performed well, with the latter possessing the highest sensitivity and specificity. Despite the reluctance of some laboratories in implementing the Etest macromethod due to its non-standardized format, the participating laboratories in this study were able to employ it with very few difficulties.

\section{Conclusion}

The debate on the clinical significance of GISA, and in particular hGISA is likely to continue for the foreseeable future $[1,6,7]$. However, some studies clearly demonstrate that h-GISA is associated with poorer clinical outcome and longer hospitalization [17]. The implementation of non-standardized methods to detect relatively rare and unusual forms of resistance (i.e. GISA and hGISA) must be ratified using multi-center data carried out under blind conditions such as those outlined in this study. The clinical significance of GISA and particularly hGISA can only be truly assessed by first employing appropriate methods to detect it. 


\section{Acknowledgements}

This work was supported by a grant of Pharmacia and logistical support of Fornix, The Netherlands. Thanks to Ton Smeets for his continuing support and to Mandy Wootton for characterization of the MRSA strains.

\section{References}

I. Arakawa $Y$, Ike $Y$, Nagasawa M: Where has vancomycin-heterogeneously resistant Staphylococcus aureus gone? Lancet 2004, 363: |40I.

2. Fridkin SK, Hageman J, McDougal LK, Mohammed J, Jarvis WR, Perl TM, Tenover TC: Epidemiological and microbiological characterization of infections by Staphylococcus aureus with reduced susceptibility to vancomycin, United States I997200 I. Clin Infect Dis 2003, 36:429-39.

3. Gemmell CG: Glycopeptide resistance in Staphylococcus aureus: is it a real threat? J Infect Chemother 2004, I 0:69-75.

4. Goldstein FW, Kitzis MD: Vancomycin-resistant Staphylococcus aureus: no apocalypse now. Clin Microbial Infect 2003, 9:76I-765.

5. Hiramatsu K, Hanaki K, Ino T, Yabuta K, Oguri T, Tenover FC: Methicillin-resistant Staphylococcus aureus clinical strain with reduced vancomycin susceptibility. J Antimicrob Chemother 1997, 40:। 35-6.

6. Howe RA, Monk A, Wootton M, Walsh TR, Enright MC: Vancomycin susceptibility within methicillin-resistant Staphylococcus aureus lineages. Emerg Infect Dis 2004, 10:855-857.

7. Howe RA, Walsh TR: hGISA: seek and you shall find. Lancet 2004, 364:500-50I.

8. Walsh TR, Howe RA: The prevalence and mechanisms of vancomycin resistance in Staphylococcus aureus. Ann Review Microbiol 2002, 56:657-75.

9. Smith TL, Pearson ML, Wilcox KR, Cosme Cruz PH, Lancaster MV, Robinson-Dunn B, Tenover FC, Zervos MJ, Band JD, White E, Jarvis WR: Emergence of vancomycin resistance in Staphylococcus aureus. N Engl J Med 1999, 340:493-501.

10. Chang S, Sievert DM, Hageman JC, Bolton HL, Tenover FC, Downes FP, Shah S, Rudrik JT, Pupp GR, Brown WJ, Cardo S, Fridkin SK: Infection with vancomycin-resistant Staphylococcus aureus containing the van resistance gene. N Eng J Med 2003, 348: I342-7.

II. Tenover FC, Weigel LM, Applebaum PC, McDougal LK, Chaitram J, McAllister S, Clark N, Killgore G, O'Hara CM, Je L, Patel JB, Bozdogan B: Vancomycin-resistant Staphylococcus aureus isolated from a patient in Pennsylvania. Antimicrob Agents Chemother 2004, 48:275-80.

12. MacGowan AP, Wise R: Establishing MIC breakpoints and the interpretation on in-vitro susceptibility tests. BSAC News letter 2004:p48-7I.

13. National Committee for Clinical Laboratory Standards Methods for dilution antimicrobial susceptibility tests for bacteria that grow aerobically. In Approved Standard M7-A6 Sixth edition. NCCLS, Villanova, PA.; 2003.

14. Cui L, Ma X, Sato K, Okuma K, Tenover FC, Mamizuka EM, Gemmell CG, Kim MN, Ploy M-C, El-Solh N, Ferraz V, Hiramatsu K: Cell wall thickening is a common feature of vancomycin resistance in Staphylococcus aureus. J Clin Microbiol 2003, 4 I:5-I 4.

15. Hanaki H, Labischinksi H, Inaba Y, Kondo N, Murakami H, Hiramatsu $\mathrm{K}$ : Increase in glutamine-non-amidated muropeptides and peptdioglycan of vancomycin-resistant Staphylococcus aureus strain, Mu50. J Antimicrob Chemother 1998, 42:315-20.

16. Tenover FC, Lancaster MV, Hill B, Steward C, Socker S, Hancock G, O'Hara C, Clark N, Hiramatsu K: Characterization of staphylococci with reduced susceptibilities to vancomycin and other glycopeptides. J Clin Microbiol 1998, 36:1020-1027.

17. Charles PGB, Ward PB, Johnson DPR, Howden BP, Grayson ML: Clinical features associated with bacteraemia due to heterogeneous vancomycin-intermediate Staphylococcus aureus. Clin Infect Dis 2004, 38:448-5I.

18. Howe RA, Wootton M, Walsh TR, Bennett PM, MacGowan AP: Heterogeneous resistance to vancomycin in Staphylococcus aureus. J Antimicrob Chemother 2000, 45: 130.

19. Hubert SK, Mohammed JM, Fridkin SK, Gaynes RP, McGowan JE, Tenover FC: Glycopeptide-Intermediate Staphylococcus aureus: Evaluation of a novel screening method and results of a survey of selected U.S. hospitals. J Clin Microbiol 1999, 37:3590-3593.
20. Walsh TR, Bolmström A, Qwarnström A, Ho P, Wootton M, Howe RA, MacGowan AP, Diekema D: Evaluation of current methods for detection of staphylococci with reduced susceptibility to glycopeptides. J Clin Microbiol 200I, 39:2439-44.

21. Wootton M, MacGowan AP, Walsh TR, Howe RA: A multicenter study evaluating the current strategies for isolating Staphylococcus aureus strains with reduced susceptibility to glycopeptides. Journal of Clin Mic 2007, 45:329-332.

22. Wootton M, Howe RA, Hillman R, Walsh TR, Bennett PM, MacGowan AP: A modified population analysis profile method to detect Staphylococcus aureus with decreased susceptibilities to vancomycin in a UK hospital. J Antimicrob Chemother 2001, 47:399-404.

23. El Sohl N, Davi M, Morvan A, Damon HA, Marty N, GISA Group, RAISIN subgroup: Characteristics of French methicillin-resistant Staphylococcus aureus isolates with decreased susceptibility or resistance to glycopeptides. J Antimicrob Chemother 2003, 52:691-4.

24. SPSS [http://spss.com]
Publish with Biomed Central and every scientist can read your work free of charge

"BioMed Central will be the most significant development for disseminating the results of biomedical research in our lifetime. "

Sir Paul Nurse, Cancer Research UK

Your research papers will be:

- available free of charge to the entire biomedical community

- peer reviewed and published immediately upon acceptance

- cited in PubMed and archived on PubMed Central

- yours - you keep the copyright

Submit your manuscript here:

http://www.biomedcentral.com/info/publishing_adv.asp
BioMedcentral 